\title{
Desde la Admisión Hasta el Ejercicio Profesional. Una Revisión de las Carreras de Odontología en Chile
}

\author{
From Admission to Professional Practice, A Review of Dental Programs in Chile
}

Manuel Arellano-Villalón ${ }^{1,3}$ \& Ramón Fuentes ${ }^{1,2}$

ARELLANO-VILLALÓN, M. \& FUENTES, R. Desde la admisión hasta el ejercicio profesional. Una revisión de las carreras de Odontología en Chile. Int. J. Odontostomat., 14(4):623-631, 2020.

RESUMEN: Al año 1991 sólo existían tres carreras de odontología en Chile: Universidad de Chile (1911), Universidad de Concepción (1919) y la Universidad de Valparaíso (1955 como sede de la U. de Chile). Actualmente, existen 21 universidades que dictan un total de 32 carreras de odontología, titulando aproximadamente 1500 nuevos profesionales cada año, lo cual, aparte de disparar las cifras de profesionales en nuestro sistema, levanta la duda de cuantos estudiantes se encuentran en formación y cuál es el perfil de admisión que se establece en estas instituciones. El objetivo de este artículo es conocer las cifras de los procesos de admisión, matrícula financiamiento y acreditación universitarios y discutirlas en base a la cantidad de profesionales habilitados para trabajar en nuestro sistema para así reflejar la situación actual que vive la Odontología en Chile. Se realizó un estudio descriptivo de corte transversal realizado en base a la revisión de las bases estadísticas públicas. A pesar de sus altos costos asociados a la implementación de espacios y materiales, la carrera sigue siendo altamente postulada. Los nuevos ingresos rondan en alrededor de 2.200 para primer año, llegando aproximadamente a 14.300 estudiantes en todos los niveles para el 2019. El Estado de Chile debería abordar los problemas que se desprenden de estos indicadores, a través de una discusión seria y sistemática, incorporando diversos actores y basándose en información sobre la real necesidad de atención en salud de la población y la disponibilidad estructural del sistema de salud público y privado para soportar determinado número de profesionales. La apertura de nuevas carreras de odontología se visualiza como un problema, mientras el país avanza a pasos lentos en una regulación que es requerida de manera urgente.

PALABRAS CLAVE: Carrera de Odontología; Educación; Acreditación; Empleabilidad; Sobreoferta Laboral.

\section{INTRODUCCIÓN}

Hasta el año 1991 sólo existían tres carreras de odontología en Chile: Universidad de Chile (1911), Universidad de Concepción (1919) y la Universidad de Valparaíso (1955 como sede de la U. de Chile). La apertura Odontología en la Universidad de La Frontera marca un hito, siendo la primera carrera de odontología en abrir desde el retorno a la democracia en Chile. Es desde ahí, que comenzó la apertura de la carrera en varias casas de estudios, pasando por un alto nivel de apertura a inicios de la década del 2001-2010.

Desde la promulgación del Decreto $\mathrm{N}^{0} 2$ del 3 de enero de 1981, el régimen militar de Chile abrió la educación superior al libre mercado permitiendo la apertura de instituciones de educación superior de carácter privado sin una orientación pública bajo la premisa del libre mercado en la educación (Cruz-Coke, 2004). Esto permitió que en el año 1997 se marcara un nuevo hito en la enseñanza de la odontología, con la apertura de la primera carrera de odontología en la Universidad Mayor, una institución privada no perteneciente al G9, corporación de derecho privado sin fines de lucro, que agrupa a las universidades públicas no estatales. Actualmente, existen 21 universidades que dictan un total de 32 carreras de odontología (Tabla I), titulando aproximadamente 1500 nuevos profesionales cada año, lo cual, aparte de disparar las cifras de profesionales en nuestro sistema, levanta la duda de cuantos estudiantes se encuentran en formación y cuál es el perfil de admisión que se establece en estas instituciones.

\footnotetext{
${ }^{1}$ Departamento de Odontología Integral Adultos, Facultad de Odontología, Universidad de La Frontera, Temuco, Chile.

${ }^{2}$ Centro de Investigación en Ciencias Odontológicas (CICO), Facultad de Odontología, Universidad de La Frontera, Temuco, Chile.

${ }^{3}$ Programa de Magister en Odontología, Facultad de Odontología, Universidad de La Frontera, Temuco, Chile.
} 
Uno de los problemas detectados por algunos autores en el sistema de salud chileno fue el constante aumento de la oferta en formación de cirujanos dentistas (Cousiño, 2013), la precarización laboral (Palavecino, 2014) y la poca información existente sobre la cantidad de estos profesionales en el país. La reforma sanitaria de Chile permitió crear un registro nacional de prestadores individuales de salud (Moscoso-Matus et al., 2016) que, actualmente, es el registro más confiable para conocer la cantidad de cirujanos dentistas en nuestro país. Este registro se presenta a través de la estadística de prestadores individuales en salud elaborado por la Unidad de Registro de la Intendencia de Prestadores de salud de la Superintendencia de Salud de Chile que data del año 2009, donde al 31 de marzo de 2019 (Ministerio de Salud, 2019) informa un total de 24.530 cirujanos dentistas registrados. Al cruzar esta cifra con el total de habitantes arrojado por el CENSO 2017 da como resultado un cirujano dentista por cada 716 habitantes o 2,78 cirujanos dentista por cada 2000 habitantes, cifra que supera la planteada por la Organización Mundial de la Salud (OMS) en su reporte anual, donde indica como "insuficiente atención en salud" una cifra menor a 1 cirujano dentista por cada 2000 habitantes (World Health Organization, 2019); esto al sumarse con las mejoras relacionadas a los hitos principales que han marcado los avances en salud bucal del país se podría relacionar a un mejoramiento en indicadores de salud bucal de la población, sin embargo, algunos autores lo plantean como una sobreoferta laboral que no necesariamente se puede relacionar a la mejora de indicadores (Monsalves, 2012; Cartes-Velásquez, 2013).

El objetivo de este artículo fue conocer las cifras de los procesos de admisión, matrícula financia-miento y acreditación universitarios y discutirlas en base a la cantidad de profesionales habilitados para trabajar en nuestro sistema para así reflejar la situación actual que vive la Odontología en Chile.

\section{MATERIAL Y MÉTODO}

Estudio descriptivo de corte transversal realizado en base a la revisión de las bases estadísticas del consejo nacional de educación (CNED), plataforma MiFuturo.cl del Ministerio de Educación (MINEDUC) de Chile e 
Tabla II. Acreditación Institucional de las Universidades que imparten la carrera de Odontología y su clasificación. Revisado el 27 de noviembre de 2019.

\begin{tabular}{lccc}
\hline \multicolumn{1}{c}{ Universidad } & $\begin{array}{c}\text { Años Acreditación } \\
\text { Institucional }\end{array}$ & Áreas de Acreditación & Clasificación* \\
\hline P. U. Católica de Chile & 7 & 5 & G9 \\
U. de Chile & 7 & 5 & Estatal \\
U. de Concepción & 7 & 5 & G9 \\
U. Austral de Chile & 6 & 5 & G9 \\
U. de La Frontera & 6 & 5 & Estatal \\
U. de Talca & 6 & 5 & Estatal \\
U. de Los Andes & 5 & 5 & Privada \\
U. de Valparaíso & 5 & 5 & Estatal \\
U. Diego Portales & 5 & 5 & Privada \\
U. Andrés Bello & 5 & 4 & Privada \\
U. de Antofagasta & 5 & 4 & Estatal \\
U. del Desarrollo & 5 & 4 & Privada \\
U. Mayor & 5 & 3 & Privada \\
U. San Sebastián & 5 & 3 & Privada \\
U. Autónoma de Chile & 4 & 4 & Privada \\
U. de La Serena & 4 & 4 & Estatal \\
U. Arturo Prat & 4 & 3 & Estatal \\
U. Finis Terrae & 4 & 3 & Privada \\
U. de Viña del Mar* & 4 & 2 & Privada \\
U. Bolivariana & No Acreditada & No Acreditada & Privada \\
U. Pedro de Valdivia & No Acreditada & No Acreditada & Privada \\
\hline
\end{tabular}

*Privada: a partir de la ley general de universidades privadas de 1981. G9: Universidades Tradicionales No Estatales. ${ }^{* *}$ Posee nueva decisión de acreditación por 3 años que rige desde el 09 de diciembre de 2019.

Tabla III. Número de vacantes y matriculados por carrera de odontología en proceso admisión 2019. Índices de Educación Superior de Consejo Nacional de Educación.

\begin{tabular}{|c|c|c|c|c|c|}
\hline Universidad & Sede & $\begin{array}{l}\text { Vacantes } \\
2019\end{array}$ & $\begin{array}{c}\text { Matrícula } \\
\text { primer año } \\
\text { hombres } \\
2019\end{array}$ & $\begin{array}{c}\text { Matrícula } \\
\text { primer año } \\
\text { mujeres } \\
2019\end{array}$ & $\begin{array}{l}\text { Matrícula } \\
\text { Primer Año } \\
2019\end{array}$ \\
\hline P. U. Católica de Chile & Santiago & 60 & 16 & 54 & 70 \\
\hline U. Andrés Bello & Viña del Mar & 156 & 35 & 91 & 126 \\
\hline U. Andrés Bello & Concepción & 71 & 28 & 40 & 68 \\
\hline U. Andrés Bello & Santiago & 173 & 65 & 140 & 205 \\
\hline U. Arturo Prat & Iquique & 45 & 18 & 35 & 53 \\
\hline U. Austral de Chile & Valdivia & 65 & 24 & 42 & 66 \\
\hline \multirow{2}{*}{ U. Autónoma de Chile } & Santiago & 55 & 12 & 44 & 56 \\
\hline & Temuco & 60 & 21 & 35 & 56 \\
\hline U. Bolivariana & Concepción & 30 & 3 & 4 & 7 \\
\hline U. de Ant ofagasta & Antofagasta & 40 & 15 & 41 & 56 \\
\hline U. de Chile & Santiago & 121 & 40 & 80 & 120 \\
\hline U. de Concepción & Concepción & 80 & 30 & 54 & 84 \\
\hline U. de La Frontera & Temuco & 70 & 30 & 48 & 78 \\
\hline U. de La Serena & La Serena & 60 & 29 & 34 & 63 \\
\hline U. de Los Andes & Santiago & 68 & 20 & 66 & 86 \\
\hline U. de Talca & Talca & 75 & 26 & 53 & 79 \\
\hline U. de Valparaíso & Valparaíso & 72 & 22 & 53 & 75 \\
\hline U. de Viña Del Mar & Viña del Mar & 70 & 16 & 42 & 58 \\
\hline \multirow{2}{*}{ U. del Desarrollo } & Concepción & 95 & 20 & 43 & 63 \\
\hline & Santiago & 80 & 27 & 48 & 75 \\
\hline U. Diego Portales & Santiago & 60 & 21 & 46 & 67 \\
\hline \multirow[t]{2}{*}{ U. Finis Terrae } & Santiago & 40 & 10 & 31 & 41 \\
\hline & Temuco & 55 & 8 & 17 & 25 \\
\hline \multirow[t]{3}{*}{ U. Mayor } & Santiago & 100 & 32 & 55 & 87 \\
\hline & Antofagasta & \multicolumn{4}{|c|}{ Sin Admisión 2019.} \\
\hline & La Serena & 25 & 11 & 20 & 31 \\
\hline \multirow[t]{3}{*}{ U. Pedro de Valdivia } & Santiago & \multicolumn{4}{|c|}{ Sin Admisión 2019.} \\
\hline & Chillán & \multicolumn{4}{|c|}{ Sin Admisión 2019.} \\
\hline & Puerto Montt & 60 & 13 & 36 & 49 \\
\hline \multirow{3}{*}{ U. San Sebastián } & Valdivia & 60 & 19 & 22 & 41 \\
\hline & Concepción & 120 & 36 & 83 & 119 \\
\hline & Santiago & 130 & 57 & 122 & 179 \\
\hline TOTAL & & 2196 & 704 & 1479 & 2183 \\
\hline
\end{tabular}


información publicada en páginas web de instituciones de educación superior en Chile. Se utiliza la estadística del año 2019 de la división de educación superior del CNED en la cual se muestra datos de matrícula, vacantes, aranceles y puntajes de ingreso de las instituciones que imparten la carrera de odontología en Chile. En caso de no encontrar algún dato, se consulta a la página web de cada institución.

La información sobre el proceso de gratuidad fue adquirida desde la plataforma web www.gratuidad.cl dispuesta por el gobierno de Chile a través de la Subsecretaría de educación superior del MINEDUC.
Los registros de las carreras cerradas fueron adquiridos en CNED y a través de entrevistas personales con egresados de la carrera para el caso de la Universidad San Sebastián sede Osorno.

La revisión final de la información se hizo entre el 25 y 29 de noviembre de 2019 por un solo investigador (MAV).

\section{RESULTADOS}

Los resultados son presentados a través de las Tablas I a VIII.

Tabla IV. Matricula total de estudiantes de odontología año 2019. Índices de Educación Superior de Consejo Nacional de Educación 2019.

\begin{tabular}{|c|c|c|c|c|}
\hline Universidad & Sede & $\begin{array}{c}\text { Matríc ula total } \\
\text { hombres } \\
2019\end{array}$ & $\begin{array}{l}\text { Matrícula total } \\
\text { mujeres } \\
2019\end{array}$ & $\begin{array}{c}\text { Matrícula Total } \\
2019\end{array}$ \\
\hline \multirow[t]{2}{*}{ P. U. Católica de Chile } & Santiago & 122 & 319 & 441 \\
\hline & Viña del Mar & 206 & 474 & 680 \\
\hline \multirow[t]{2}{*}{ U. Andrés Bello } & Concepción & 108 & 188 & 296 \\
\hline & Santiago & 413 & 767 & 1180 \\
\hline U. Arturo Prat & Iquique & 93 & 172 & 265 \\
\hline U. Austral de Chile & Valdivia & 161 & 238 & 399 \\
\hline U. Autónoma de Chile & Santiago & 136 & 284 & 420 \\
\hline U. Autónoma de Chile & Temuco & 109 & 202 & 311 \\
\hline U. Bolivariana & Concepción & 24 & 17 & 41 \\
\hline U. de Antofagasta & Antofagasta & 143 & 248 & 391 \\
\hline U. de Chile & Santiago & 302 & 478 & 780 \\
\hline U. de Concepción & Concepción & 189 & 330 & 519 \\
\hline U. de La Frontera & Temuco & 166 & 280 & 446 \\
\hline U. de La Serena & La Serena & 124 & 217 & 341 \\
\hline U. de Los Andes & Santiago & 149 & 390 & 539 \\
\hline U. de Talca & Talca & 176 & 381 & 557 \\
\hline U. de Valparaíso & Valparaíso & 145 & 341 & 486 \\
\hline U. de Viña del Mar & Viña del Mar & 213 & 354 & 567 \\
\hline \multirow{2}{*}{ U. del Desarrollo } & Concepción & 181 & 363 & 544 \\
\hline & Santiago & 173 & 348 & 521 \\
\hline U. Diego Portales & Santiago & 175 & 297 & 472 \\
\hline U. Finis Terrae & Santiago & 129 & 317 & 446 \\
\hline \multirow{3}{*}{ U. Mayor } & Temuco & 82 & 160 & 242 \\
\hline & Santiago & 207 & 383 & 590 \\
\hline & La Serena & 58 & 93 & 151 \\
\hline \multirow{2}{*}{ U. Pedro de Valdivia } & Antofagasta* & 9 & 24 & 33 \\
\hline & Chillán* & 15 & 26 & 41 \\
\hline \multirow{5}{*}{ U. San Sebastián } & Santiago* & 23 & 31 & 54 \\
\hline & Puerto Montt & 129 & 217 & 346 \\
\hline & Valdivia & 113 & 176 & 289 \\
\hline & Concepción & 326 & 521 & 847 \\
\hline & Santiago & 383 & 693 & 1076 \\
\hline \multicolumn{2}{|c|}{ Total } & 4982 & 9329 & 14311 \\
\hline
\end{tabular}

${ }^{*}$ Carreras sin admisión 2019. 
ARELLANO-VILLALÓN, M. \& FUENTES, R. Desde la admisión hasta el ejercicio profesional. Una revisión de las carreras de Odontología en Chile. Int. J. Odontostomat., 14(4):623-631, 2020.

Tabla V. Acreditación carreras de odontología de Chile. Histórico Comisión Nacional de Acreditación de Pregrado (CNAP) y Comisión Nacional de Acreditación (CNA-Chile).

\begin{tabular}{|c|c|c|c|}
\hline Universidad & Acreditación Actual $^{*}$ & Ente Acreditador (última acreditación) ${ }^{* *}$ & $\begin{array}{l}\text { Acreditación Procesos } \\
\text { Anteriores }\end{array}$ \\
\hline P. U. Católica de Chile & 6 años $(2018-2024)$ & Agencia Acreditadora: A\&C / CNA-Chile & No Tiene \\
\hline U. Andrés Bello & 6 años $(2018-2024)$ & Agencia Acreditadora: ADC / CNA-Chile & $\begin{array}{l}4 \text { años }(2011-2015) \\
3 \text { años (2015-2018) }\end{array}$ \\
\hline U. Arturo Prat & 3 años (2019-2022) & CNA-Chile & No Tiene \\
\hline U. Austral de Chile & 4 años $(2019-2023)$ & CNA-Chile & 5 años (2014-2019) \\
\hline $\begin{array}{l}\text { U. Autónoma de Chile } \\
\text { U. Bolivariana }\end{array}$ & 5 años $(2018-2023)$ & $\begin{array}{l}\text { Agencia Acreditadora: ADC } \\
\text { No Acreditada }\end{array}$ & $\begin{array}{l}\text { No Tiene } \\
\text { No Tiene }\end{array}$ \\
\hline U. de Antofagasta & 6 años $(2015-2021)$ & Agencia Acreditadora: Acreditacción & $\begin{array}{l}2 \text { años }(2006-2008) \\
4 \text { años }(2010-2014)\end{array}$ \\
\hline $\begin{array}{l}\text { U. de Chile } \\
\text { U. de Concepción }\end{array}$ & $\begin{array}{l}7 \text { años }(2013-2020) \\
7 \text { años }(2012-2019)\end{array}$ & $\begin{array}{l}\text { Agencia Acreditadora: AACS } \\
\text { Agencia Acreditadora: Akredita }\end{array}$ & $\begin{array}{l}6 \text { años }(2006-2012) \\
6 \text { años }(2005-2011)\end{array}$ \\
\hline U. de La Frontera & 6 años $(2018-2024)$ & Agencia Acreditadora: Qualitas / CNA-Chile & $\begin{array}{l}4 \text { años }(2006-2010) \\
5 \text { años }(2012-2017)\end{array}$ \\
\hline $\begin{array}{l}\text { U. de La Serena } \\
\text { U. de Los Andes }\end{array}$ & $\begin{array}{c}\text { No Acreditada (En proceso) } \\
7 \text { años }(2018-2025)\end{array}$ & $\begin{array}{l}\text { En proceso de acreditación con CNA-Chile } \\
\text { Agencia Acreditadora: A\&C / CNA-Chile }\end{array}$ & $\begin{array}{c}\text { No Tiene } \\
6 \text { años }(2012-2018)\end{array}$ \\
\hline U. de Talca & 5 años $(2018-2023)$ & Agencia Acreditadora: A\&C & $\begin{array}{l}4 \text { años }(2005-2009) \\
5 \text { años }(2012-2017)\end{array}$ \\
\hline U. de Valparaíso & 7 años $(2015-2022)$ & Agencia Acreditadora: Acreditacción & $\begin{array}{l}5 \text { años (2004-2009) } \\
5 \text { años (2010-2015) }\end{array}$ \\
\hline U. de Viña del Mar & & No Acreditada & No Tiene \\
\hline U. del Desarrollo & 5 años $(2015-2020)$ & Agencia Acreditadora: ADC & No Tiene \\
\hline U. Diego Portales & 6 años $(2017-2023)$ & Agencia Acreditadora: Qualitas & 4 años (2012-2016) \\
\hline U. Finis Terrae & 6 años $(2018-2024)$ & Agencia Acreditadora: Acreditacción & $\begin{array}{l}4 \text { años }(2010-2014) \\
3 \text { años }(2015-2018)\end{array}$ \\
\hline U. Mayor & 6 años $(2018-2024)$ & Agencia Acreditadora: ADC / CNA-Chile & $\begin{array}{l}5 \text { años (2008-2013) } \\
5 \text { años (2013-2018) }\end{array}$ \\
\hline U. Pedro de Valdivia & & No Acreditada & No Tiene \\
\hline U. San Sebastián & 7 años (2015-2022) & Agencia Acreditadora: ADC & 3 años (2012-2015) \\
\hline
\end{tabular}

* Consulta en buscador avanzado Comisión Nacional de Acreditación CNA-Chile. 27 noviembre 2019. **El 21 de noviembre de 2019 se promulga la "ley corta de educación superior" (ley $N^{\circ} 21.186$ ) la cual modifica el párrafo $5^{\circ}$ de las disposiciones generales de la ley $N^{\circ} 21.091$ sobre Educación Superior; lo anterior permite que las carreras que iniciaron procesos de acreditación desde el 29 de mayo de 2018 con agencias, puedan terminarlos con las mismas.

Tabla VI. Adscripción a gratuidad y al sistema único de admisión y aranceles de carreras de odontología expresados en pesos chilenos. Año 2019.

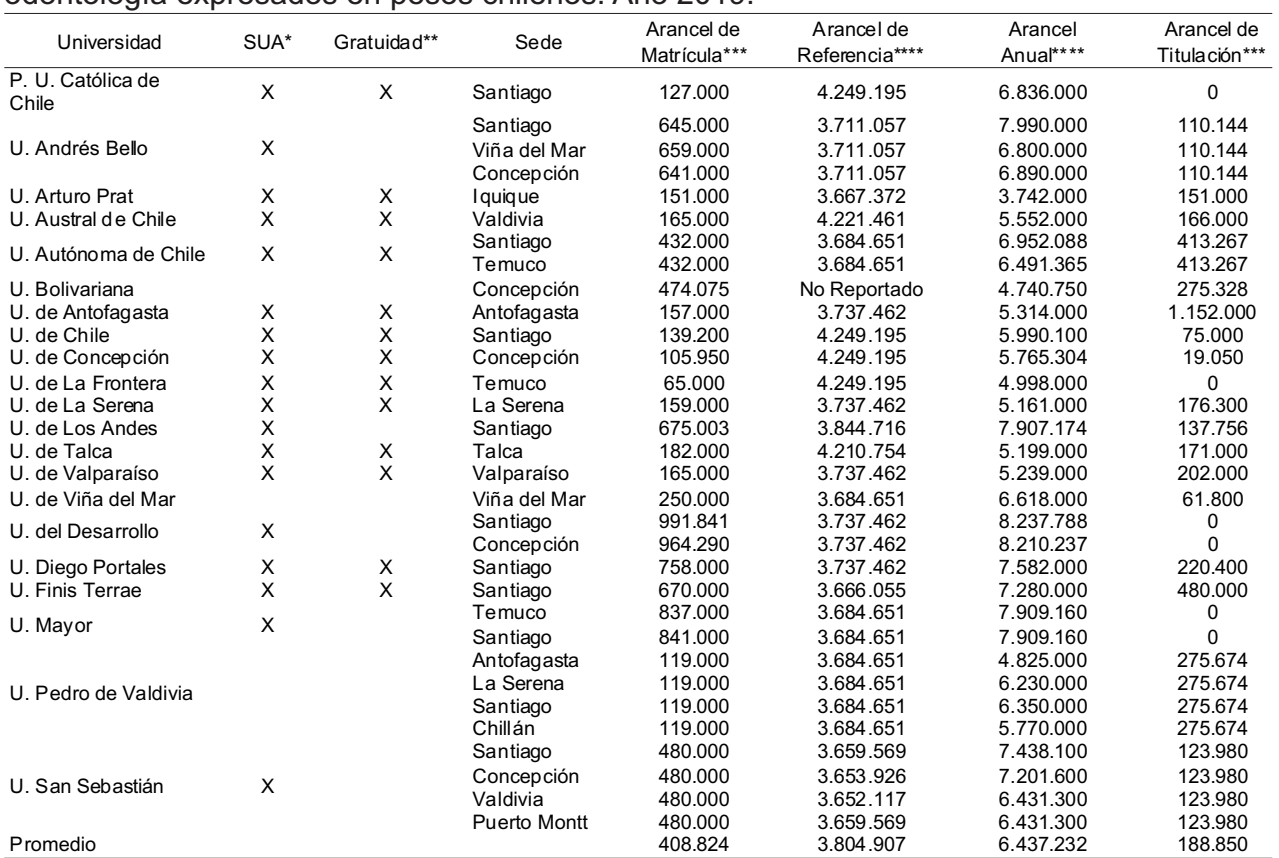

* Departamento de Evaluación, Medición y Registro Educacional - DEMRE, Universidad de Chile. Consulta página web psu.demre.cl el 21 de noviembre de 2019. **Resolución exenta № 159 del 17 de enero de 2019. División de Educación Superior, Ministerio de Educación, Gobierno de Chile. Disponible en: www.gratuidad.cl consultada el 21 de noviembre de 2019. *** Índices de Educación Superior de Consejo Nacional de Educación 2019. Disponible en: www.cned.cl consultada el 21 de noviembre de 2019. ${ }^{* * *}$ Aranceles de referencia universidades 2019. Portal de beneficios estudiantiles, Ministerio de Educación, Gobierno de Chile. 
Tabla VII. Puntaje PSU Promedio y Puntaje Ponderado admisión 2019 carreras de odontología de universidades adscritas al SUA. Consejo Nacional de Educación.

\begin{tabular}{|c|c|c|c|c|c|c|c|}
\hline Universidad & Sede & $\begin{array}{l}\text { Máximo } \\
\text { Promedio } \\
\text { PSU* }^{*}\end{array}$ & $\begin{array}{c}\text { Promedi } \\
\text { o PSU* }\end{array}$ & $\begin{array}{l}\text { Mínimo } \\
\text { Promedio } \\
\text { PSU* }^{*}\end{array}$ & $\begin{array}{c}\text { Puntaje } \\
\text { Ponderado } \\
\text { Primer } \\
\text { Seleccionado* }\end{array}$ & $\begin{array}{c}\text { Puntaje } \\
\text { Ponderad } \\
\text { o } \\
\text { Promedio* }\end{array}$ & $\begin{array}{c}\text { Puntaje } \\
\text { Ponderado } \\
\text { Último } \\
\text { Seleccionado* }\end{array}$ \\
\hline $\begin{array}{l}\text { P. U. Católica de } \\
\text { Chile }\end{array}$ & Santiago & 749 & 691 & 592 & 772 & 727 & 707 \\
\hline \multirow{3}{*}{ U. Andrés Bello } & Viña del Mar & 712 & 577 & 500 & 714 & 604 & 501 \\
\hline & Concepción & 667 & 559 & 503 & 677 & 585 & 509 \\
\hline & Santiago & 683 & 573 & 500 & 710 & 593 & 525 \\
\hline U. Arturo Prat & Iquique & 694 & 566 & 501 & 705 & 619 & 556 \\
\hline U. Austral de Chile & Valdivia & 731 & 600 & 500 & 748 & 648 & 607 \\
\hline \multirow{2}{*}{$\begin{array}{l}\text { U. Autónoma de } \\
\text { Chile }\end{array}$} & Santiago & 664 & 604 & 551 & 678 & 614 & 552 \\
\hline & Temuco & 707 & 593 & 550 & 702 & 594 & 552 \\
\hline U. de Antofagasta & Antofagasta & 684 & 587 & 479 & 733 & 647 & 557 \\
\hline U. de Chile & Santiago & 742 & 677 & 598 & 765 & 708 & 689 \\
\hline U. de Concepción & Concepción & 729 & 661 & 593 & 736 & 701 & 678 \\
\hline U. de La Frontera & Temuco & 755 & 637 & 543 & 733 & 679 & 645 \\
\hline U. de La Serena & La Serena & 763 & 627 & 510 & 778 & 672 & 640 \\
\hline U. de Los Andes & Santiago & 718 & 654 & 572 & 751 & 688 & 661 \\
\hline U. de Talca & Talca & 720 & 630 & 510 & 748 & 695 & 660 \\
\hline U. de Valparaíso & Valparaíso & 757 & 640 & 563 & 763 & 689 & 660 \\
\hline \multirow{2}{*}{ U. del Desarrollo } & Concepción & 735 & 605 & 510 & 733 & 606 & 510 \\
\hline & Santiago & 733 & 644 & 565 & 730 & 650 & 602 \\
\hline U. Diego Portales & Santiago & 707 & 646 & 587 & 691 & 654 & 630 \\
\hline U. Finis Terrae & Santiago & 648 & 604 & 571 & 678 & 629 & 608 \\
\hline \multirow{2}{*}{ U. Mayor } & Temuco & 703 & 590 & 504 & 739 & 619 & 562 \\
\hline & Santiago & 694 & 607 & 495 & 715 & 623 & 580 \\
\hline \multirow{4}{*}{ U. San Sebastián } & Puerto Montt & 698 & 554 & 456 & 723 & 578 & 485 \\
\hline & Valdivia & 680 & 532 & 467 & 710 & 561 & 478 \\
\hline & Concepción & 687 & 561 & 464 & 743 & 603 & 510 \\
\hline & Santiago & 672 & 555 & 471 & 648 & 559 & 515 \\
\hline
\end{tabular}

*El indicador "Promedio PSU" refiere al promedio de los puntajes de las pruebas obligatorias de Lenguaje y Comunicación y Matemáticas. El indicador es igual para todas las universidades. ${ }^{* *}$ El indicador "Puntaje Ponderado" refiere al puntaje con el que el estudiante postula a la admisión de una institución de educación superior y varía según las ponderaciones qué de ésta a las pruebas obligatorias, pruebas electivas, notas de enseñanza media (NEM) y ranking con los que se presenta el estudiante.

\section{DISCUSIÓN}

La Carrera de Odontología a pesar de los altos costos asociados a la implementación de espacios y materiales sigue siendo una carrera altamente demandada en términos de postulaciones completando en general todos los cupos ofrecidos por las instituciones que la imparten, aún cuando hay diferencias de acreditación de las instituciones que la dictan, tanto en número de años de acreditación institucional y áreas de acreditación de las mismas como en los diferentes años de acreditación de carreras de forma individual; atendiendo a las legislación vigente que dicta la obligatoriedad en esto. La ley 21.091 sobre educación superior establece un nuevo sistema de aseguramiento de la calidad en las instituciones de educación supe- rior con una acreditación integral y obligatoria promoviendo una fiscalización homogénea de la calidad de las carreras, programas, sedes y otras funciones institucionales, esta ley también incorpora a la odontología dentro de las profesiones cuya acreditación es obligatoria. Lo anterior viene a regular en alguna medida los problemas de sobreoferta laboral encontrados por algunos autores (Monsalves; Cousiño; CartesVelásquez; Palavecino; Cerón Benavides, 2017). Además, se puede apreciar que la variabilidad en el número de instituciones y carreras acreditadas ha disminuido en relación con lo reportado por otro autor desde el año 2015 (Carrizo, 2016). Es importante mantenerse alerta sobre las consecuencias a largo plazo de 
ARELLANO-VILLALÓN, M. \& FUENTES, R. Desde la admisión hasta el ejercicio profesional. Una revisión de las carreras de Odontología en Chile.

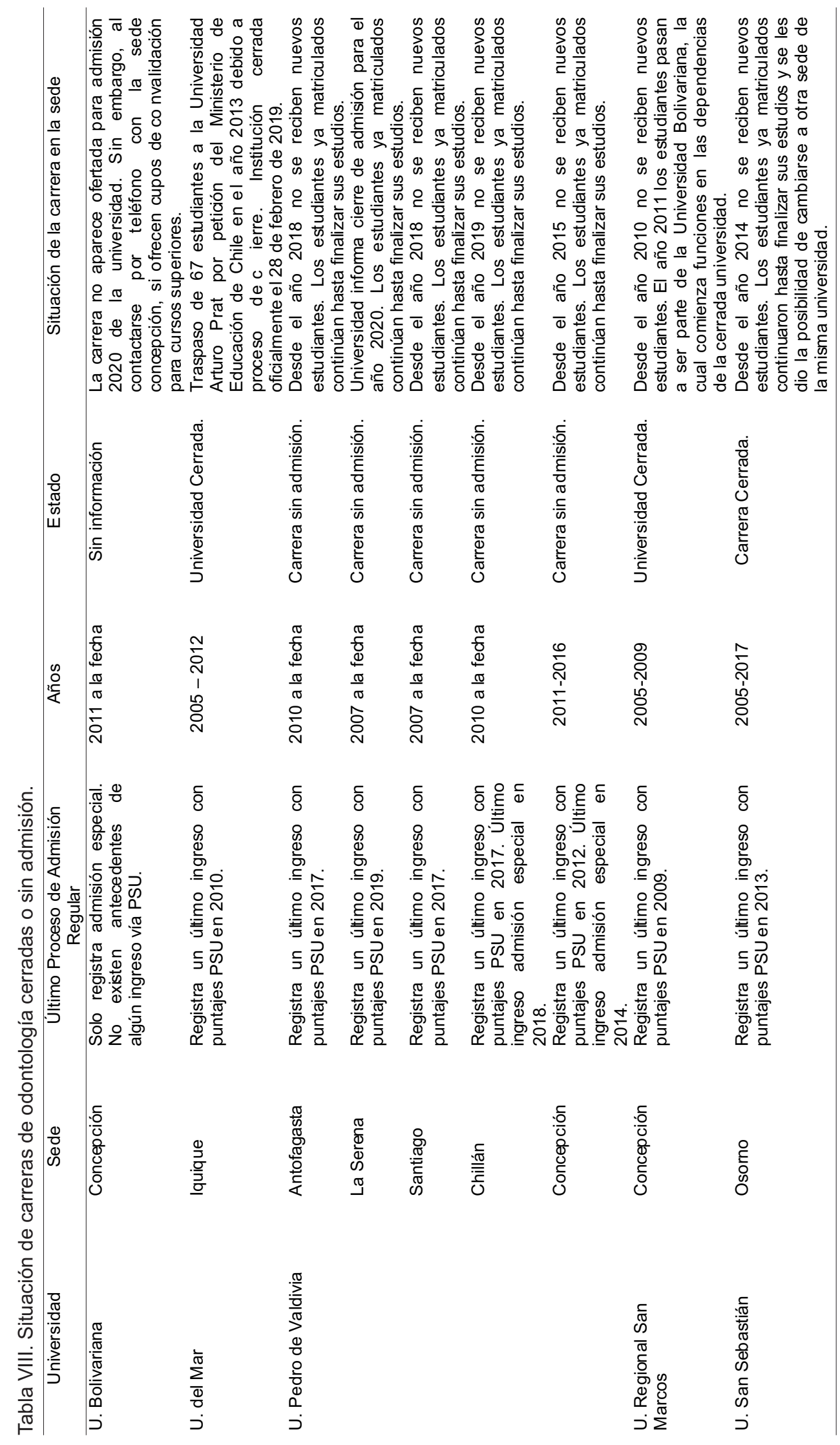

la acreditación obligatoria de la carrera de odontología, encontrándose actualmente acreditadas un total de 17 considerando que algunas casas de estudios tienen más de una sede (Tabla V).

Previo al ingreso a primer año, cabe destacar algunos puntos con respecto a la admisión, donde se puede ver la tendencia aún de mejores puntajes PSU en universidades tradicionales (Tabla VII) atendiendo a reportes de otros autores sobre un puntaje más bajo en planteles privados no tradicionales (Espinoza, 2014, 2015). Un problema detectado en esta área es que aún existen universidades no adscritas al sistema único de admisión, lo cual aporta a aumentar la heterogeneidad en criterios de ingreso para estudiantes de odontología; estas son tres: U. Bolivariana, U. Pedro de Valdivia y U. de Viña del Mar, esta última siendo la única con proceso de admisión para Odontología 2020 abierto.

El alto número de carreras de odontología en Chile es un tema recurrente en literatura gris de diversos autores. Uno de los temas a destacar en este problema es el alto número de ingresos de nuevos estudiantes de odontología, los cuales rondan en alrededor de 2.200 cada año. En 
Chile, no existe una regularización de mercado en las vacantes que puede generar una institución de educación superior, debido al sistema de libre mercado establecido para la educación hoy considerada un bien de consumo (Espinoza, 2017). Las 5 carreras de odontología con mayores ingresos a primer año son U. Andrés Bello (Santiago y Viña del Mar), U. San Sebastián (Santiago y Concepción) y la U. de Chile, sumando un total de 749 matrículas en el primer año de la carrera; esto equivale a un $34,3 \%$ de la matrícula total de primer año en Chile el año 2019 (Tabla III).

Debido a lo anterior, existe un alto número de estudiantes cursando actualmente la carrera de Odontología en Chile, llegando aproximadamente a 14.300 a marzo de 2019. Las 5 carreras de odontología con mayores matriculas totales son U. San Sebastián con 2.558, U. Andrés Bello con 2.156, U. del Desarrollo con 1.065, U. Mayor con 832 y U. de Chile con 780, considerando la sumatoria de las sedes en el caso de las cuatro primeras; esto equivale a un $51,6 \%$ de la matricula total de estudiantes de Odontología de Chile (Tabla IV).

Con respecto al financiamiento de la carrera de odontología, algunas de las instituciones que la imparten se encuentran adscritas a gratuidad (Tabla VI), política pública donde los estudiantes pertenecientes a familias dentro del $60 \%$ de menores ingresos económicos en el país, obtienen cobertura estatal para el pago de arancel y matrícula durante la duración formal de la carrera, estos montos se fijan en base a los años de acreditación de la institución (Tabla II). Un total de siete instituciones que imparten odontología (33\%) no se encuentran adscritas, lo cual adhiere un nuevo problema a la profesión, que es la deuda adquirida por el estudiante al formarse. Nuestra carrera se encuentra dentro de las mas costosas del país en termino de arancel según lo mostrado por MiFuturo.cl y plasmado en la literatura por algunos autores (Alcota et al., 2016). Esto nos da a reflexionar la real viabilidad económica de estudiar odontología en Chile y si los postulantes acceden a esta información como insumo al momento de decidirse por estudiar la profesión.

En cuanto a la titulación, el año 2019 las instituciones de educación superior entregaron al país aproximadamente 1.500 titulados, según lo informado por el Ministerio de Educación de Chile a través de la subsecretaria de educación superior en la página MiFuturo.cl, lo cual sin duda aportará a la sobreoferta laboral presente en la profesión. Esto podría contribuir en alguna medida, en base al libre mercado de nuestro país, a ofrecer un mayor acceso a la salud oral a través de una mayor competencia en el área (Monsalves), sin embargo, como contraste se puede tomar como ejemplo la Encuesta Nacional de Salud 2016-2017, donde se aprecia que el 52,8 \% de las personas no asiste al dentista hace más de un año (Ministerio de Salud, 2018) lo que se podría interpretar como una inconsistencia en la idea de mayor cantidad de dentistas es igual a mayor acceso de la población.

Otro punto a destacar sobre los titulados son las formas que se presentan para mejorar los índices de empleabilidad y aumentar así sus remuneraciones. Es de conocimiento general que cursar programas de especialización y postgrado es uno de los recursos más utilizados, sin embargo, un auge de programas de formación continua está abriendo la carta de oportunidades para los nuevos y antiguos titulados. Todo esto en función de encontrar nuevas áreas de desempeño y evitar una mayor precarización laboral con una motivación de estabilidad laboral por sobre vocación.

El estado de chile, a través sus instituciones, debería abordar los problemas que se desprenden en esta y varias otras publicaciones citadas, a través de una discusión seria y sistemática, incorporando a las universidades, organizaciones profesionales tanto políticas como científicas y basándose en información sobre la real necesidad de atención en salud oral de la población y la disponibilidad estructural del sistema de salud público y privado para soportar determinado número de profesionales. En el caso del dentista con énfasis en el requerimiento de equipamiento técnico y estructural especial para lograr la atención correcta en base a criterios sanitarios y éticos. La apertura de nuevas carreras de odontología se visualiza como un problema (Guíñez et al., 2018), mientras el país avanza a pasos lentos en una regulación que es requerida de manera urgente (Tabla VIII).

ARELLANO-VILLALÓN, M. \& FUENTES, R. From admission to professional practice, A review of dental programs in Chile. Int. J. Odontostomat., 14(4):623-631, 2020.

ABSTRACT: In 1991 there were only three dental programs in Chile: Universidad de Chile (1911), Universidad de Concepción (1919) and Universidad de Valparaíso (1955, as campus of $\mathrm{U}$. de Chile). Currently, there are 21 universities that teach a total of 32 dental programs, graduating approximately 1500 new professionals each year, which, in addition to increasing the number of dentists in our system, raises the question as to how many students are in training, 
and what is the admission profile established by these institutions. The aim of this article is to know the number of admissions, registration, financing, and university accreditation process, based on the number of qualified professionals working in our system, in order to reflect the current situation of dentistry in Chile. A descriptive cross-sectional study was carried out based on the review of the public statistical resources. Despite the high cost of the program, associated with the implementation and materials, the program has many applicants. The number of enrolled students each year in the first semester is around 2200. Therefore, a high number of students are currently enrolled in all semesters, totalling approximately 14,300 in 2019 . The Chilean government should address this problem based on information about the real need for healthcare of the population, within the framework of the public and private health system to support a certain number of professionals. The opening of new dental programs is viewed as a problem, while the country is advancing slowly in a regulation that is urgently required.

KEY WORDS: dental program, education, accreditation, employability, labor oversupply.

\section{REFERENCIAS BIBLIOGRÁFICAS}

Alcota, M.; Ruiz de Gauna, P. \& González, F. E. Dental programs in the current context of Chilean higher education. Int. J. Odontostomat., 10(1):85-91, 2016.

Carrizo, A. State of accreditation of undergraduate dental programs in Chile, 2015. J. Oral Res., 5(1):19-26, 2016.

Cartes-Velásquez, R. A. Exponential growth of dental schools in Chile: effects on academic, economic and workforce issues. Braz. Oral Res., 27(6):471-7, 2013.

Cerón Benavides, A. P. Dentistas en Chile: ¿Cómo somos, cuántos somos y cuántos necesita el país? Sitio Web. Santiago de Chile, Webdental, 2017. Disponible en: https:// webdental.wordpress.com/2017/03/24/dentistas-en-chile-comosomos-cuantos-somos-y-cuantos-necesita-el-pais/

Cousiño, S. ¿Cuántos somos actualmente los dentistas en Chile? La burbuja odontológica. Contraangulo. Sitio Web. Santiago de Chile, Revista Capítulo de Odontólogos de Libre Ejercicio de Chile, 2013. Disponible en: http://www.contraangulo.com/2013/ 07/cuantos-somos-actualmente-los-dentistas.html

Cruz-Coke, M. R. Evolución de las universidades chilenas 19812004. Rev. Med. Chile, 132(12):1543-9, 2004.

Espinoza, L. Distribution of PSU scores for dentistry schools in Chile during 2012-2014. J. Oral Res., 3(2):100-4, 2014.

Espinoza, L. Evolution of the application process to Dentistry in universities taking part in the Prueba de Selección Universitaria (PSU). Chile, 2012-2014. J. Oral Res., 4(1):51-7, 2015.

Espinoza, O. Privatización de la educación superior en Chile: consecuencias y lecciones aprendidas. EccoS Rev. Cient., (44):175202, 2017.

Guíñez, J.; Guajardo, P.; Cartes-Velásquez, R. \& Campos, V. The current status of dental education and the dental profession in Chile. Pesqui. Bras. Odontopediatria Clin. Integr., 18(1):e3875, 2018.

Ministerio de Salud (MINSAL). Encuesta Nacional de Salud 20162017. Segunda entrega de resultados. Santiago de Chile, Departamento de Epidemiología, División de Planificación Sanita- ria, Subsecretaría de Salud Pública, Ministerio de Salud, Gobierno de Chile, 2018. Disponible en: https://www.minsal.cl/wpcontent/uploads/2018/01/2 - ResultadosENS_MINSAL_31_01_2018.pdf

Ministerio de Salud (MINSAL). Estadísticas de Prestadores Individuales de Salud periodo 1 de abril de 2009 al 31 de marzo de 2019. Santiago de Chile, Unidad de Registro, Intendencia de Prestadores de Salud, Superintendencia de Salud, Ministerio de Salud Gobierno de Chile, 2019. Disponible en: http:// www.supersalud.gob.cl/664/articles-18219_recurso_1.pdf

Monsalves, M. J. La odontología que vivimos y no queremos: una crítica al escenario actual. Rev. Chil. Salud Pública, 16(3):2416, 2012.

Moscoso-Matus, K.; Vásquez, P. S. \& Botto Beytía, A. M. Odontólogos del registro nacional de prestadores individuales de salud. Rev. Clin. Periodoncia Implantol. Rehabil. Oral, 9(1):427, 2016.

Palavecino, G. P. Crisis del Sistema Sanitario, Un Ejemplo desde la Odontología en Chile. Sitio Web. Santiago de Chile, El Quinto Poder, 2014. Disponible en: https://www.elquintopoder.cl/salud/ crisis-del-sistema-sanitario-un-ejemplo-desde-la-odontologia-enchile/

World Health Organization (WHO). World Health Statistics 2019. Monitoring Health For The Sdgs, Sustainable Development Goals. Ginebra, World Health Organization, 2019. Disponible en: https://apps.who.int/iris/bitstream/handle/10665/324835/ 9789241565707-eng.pdf?ua=1

Dirección para correspondencia

Dr. Ramón Fuentes Fernández

Centro de Investigación en Ciencias Odontológicas (CICO)

Facultad de Odontología

Universidad de La Frontera

Av. Francisco Salazar 01145, Pabellón L

Temuco

CHILE

Email: ramon.fuentes@ufrontera.cl

Recibido : 09-04-2020

Aceptado: 24-06-2020 